\title{
Predictors of delayed recovery following pediatric sports-related concussion: a case-control study
}

\author{
Joseph H. Miller, MD, ${ }^{1}$ Clarence Gill, BS, ${ }^{1}$ Elizabeth N. Kuhn, MD, ${ }^{1}$ Brandon G. Rocque, MD, MS, ${ }^{1}$ \\ Joshua Y. Menendez, MD, ${ }^{1}$ Jilian A. O'Neill, MA, ${ }^{1}$ Bonita S. Agee, PhD, MPH, ${ }^{1}$ \\ Steven T. Brown, MD, ${ }^{1}$ Marshall Crowther, MD, ${ }^{2}$ R. Drew Davis, MD, ${ }^{2}$ \\ Drew Ferguson, MEd, ${ }^{2}$ and James M. Johnston, MD'
}

Departments of ${ }^{1}$ Neurosurgery and ${ }^{2}$ Sports Medicine, University of Alabama at Birmingham, Alabama

OBJECTIVE Pediatric sports-related concussions are a growing public health concern. The factors that determine injury severity and time to recovery following these concussions are poorly understood. Previous studies suggest that initial symptom severity and diagnosis of attention deficit hyperactivity disorder (ADHD) are predictors of prolonged recovery (> 28 days) after pediatric sports-related concussions. Further analysis of baseline patient characteristics may allow for a more accurate prediction of which patients are at risk for delayed recovery after a sports-related concussion.

METHODS The authors performed a single-center retrospective case-control study involving patients cared for at the multidisciplinary Concussion Clinic at Children's of Alabama between August 2011 and January 2013. Patient demographic data, medical history, sport concussion assessment tool 2 (SCAT2) and symptom severity scores, injury characteristics, and patient balance assessments were analyzed for each outcome group. The control group consisted of patients whose symptoms resolved within 28 days. The case group included patients whose symptoms persisted for more than 28 days. The presence or absence of the SCAT2 assessment had a modifying effect on the risk for delayed recovery; therefore, stratum-specific analyses were conducted for patients with recorded SCAT2 scores and for patients without SCAT2 scores. Unadjusted ORs and adjusted ORs (aORs) for an association of delayed recovery outcome with specific risk factors were calculated with logistic regression analysis.

RESULTS A total of 294 patients met the inclusion criteria of the study. The case and control groups did not statistically significantly differ in age $(p=0.7)$. For the patients who had received SCAT2 assessments, a previous history of concussion (aOR 3.67, 95\% Cl 1.51-8.95), presenting SCAT2 score < 80 (aOR 5.58, 95\% Cl 2.61-11.93), and female sex (aOR $3.48,95 \% \mathrm{Cl} 1.43-8.49$ ) were all associated with a higher risk for postconcussive symptoms lasting more than 28 days. For patients without SCAT2 scores, female sex and reporting a history of ADHD significantly increased the odds of prolonged recovery (aOR 4.41, 95\% Cl 1.93-10.07 and aOR 3.87, 95\% Cl 1.13-13.24, respectively). Concussions resulting from playing a nonhelmet sport were also associated with a higher risk for prolonged symptoms in patients with and without SCAT2 scores (OR 2.59, 95\% Cl 1.28-5.26 and OR 2.17, 95\% Cl 0.99-7.73, respectively). Amnesia, balance abnormalities, and a history of migraines were not associated with symptoms lasting longer than 28 days.

CONCLUSIONS This case-control study suggests candidate risk factors for predicting prolonged recovery following sports-related concussion. Large prospective cohort studies of youth athletes examined and treated with standardized protocols will be needed to definitively establish these associations and confirm which children are at highest risk for delayed recovery.

http://thejns.org/doi/abs/10.3171/2015.8.PEDS14332

KEY WORDS concussion; SCAT2 score; pediatric; trauma; recovery; head injury

$\mathrm{M}$ ILD traumatic brain injury is the reason why $16 \%$ of children require medical attention before the age of 10 years. ${ }^{1}$ Sports-related concussions in children represent $25 \%-50 \%$ of all concussions and are a significant public health concern. ${ }^{1,10}$ A concussion is defined as a "complex pathophysiological process affecting the brain, induced by biomechanical forces." 15 Concussions result in many symptoms and may affect a patient physically, cognitively, and emotionally. Several signs may accompany a concussion, including headaches, "feeling in a fog," emotional variability, loss of consciousness or amnesia, behavioral changes such as irritability, cogni-

ABBREVIATIONS ADHD = attention deficit hyperactivity disorder; CCCOA = Concussion Clinic at Children's of Alabama; SCAT2 = Sport Concussion Assessment Tool 2. SUBMITTED June 25, 2014. ACCEPTED August 27, 2015.

INCLUDE WHEN CITING Published online December 18, 2015; DOI: 10.3171/2015.8.PEDS14332. 
tive impairment such as slowed reaction times, or sleep disturbances. ${ }^{16}$ The postconcussive syndrome, therefore, is varied and may not be recognized without close observation after a mild traumatic brain injury. ${ }^{1}$

Previously, most pediatric patients were presumed to fully recover after a concussion, but its long-term neuropsychological impacts are poorly understood. The length of the recovery process depends on many factors, including severity of the concussion, age, presence of comorbidities, and a patient's postconcussive treatment. Most pediatric patients who incur a concussion recover within 3 weeks, whereas some experience symptoms that persist for longer than 1 month. 2,6-8,16,17

Many states have passed legislation that requires physicians to evaluate all patients after a concussion. This study was conducted in Alabama after a sports-related concussion law was passed in June of 2011. A multidisciplinary concussion clinic was instituted to medically examine and clear a large number of patients for return to play. On the basis of this experience, it became clear that the ability to predict which patients will have prolonged symptoms after a concussion would be a very useful clinical tool in determining prognosis and guiding therapy (such as physical and psychological rest periods)..$^{18}$

Although factors such as on-field dizziness, visual impairment, and migraines have been associated with longer recovery times after a sport-related concussion, ${ }^{11,12}$ other factors such as amnesia at the time of the injury, ${ }^{6,13,14}$ history of concussions,, 5 and an athlete's age at the time of the injury ${ }^{4,9,18}$ have also been postulated as possible factors that could predict longer symptom severity. ${ }^{18}$ To the best of the our knowledge, no case-control study has ever been performed in the pediatric population to objectively evaluate the risk factors associated with delayed recovery following a sports-related concussion.

\section{Methods \\ Study Design}

A single-center unmatched case-control study of pediatric sports-related concussions was performed. All patients were seen in the multidisciplinary Concussion Clinic at Children's of Alabama (CCCOA). Only children who sustained a concussion after the implementation of an institution-wide multidisciplinary concussion management program with a dedicated sports concussion clinic were included in this study. The University of Alabama at Birmingham Institutional Review Board approved the study.

All patients included in this study were seen at CCCOA between August 3, 2011, and January 23, 2013. The inclusion criteria of this study required that all the patients present within 4 weeks of their injury and had to have a dated follow-up visit more than 28 days from the time of injury. The Sport Concussion Assessment Tool 2 (SCAT2; from the Third International Consensus Conference on Concussion in $\operatorname{Sport}^{16}$ ) was used at the initial patient encounter and was not repeated for follow-up appointments. All types of sports-related concussions were included in this study except for those sustained from more severe injury mechanisms such as motor vehicle accidents. Patients with concussions from motor sports such as motocross and scooters or bikes were included in this study.

Any patient who exhibited a head injury resulting from a sport or nonsport activity and that caused a temporary loss of brain function followed by the onset of signs and symptoms of a concussion had a diagnosis of concussion and was referred to Sports Concussion Clinic for initial evaluation. In this study, the CCCOA emergency room attending physician or the Sports Concussion Clinic attending physician diagnosed concussions according to the definition outlined in the 2008 Zurich International Consensus on Concussion in Sport. ${ }^{15,16}$

For the analysis, the patients were divided into 2 groups. The case group consisted of patients with postconcussive symptoms that persisted longer than 28 days, indicating a delayed recovery outcome, and the control group comprised patients who had symptom resolution within 28 days, indicating an early recovery outcome. Symptom resolution was defined as the return of a patient to his or her preconcussion state of health with complete resolution of postconcussive symptoms. Recovery was defined as patients who were free of these symptoms both at rest and with exertion. The patient reported the time of symptom resolution at his or her last clinical follow-up. Patient demographic data, medical history, SCAT2 score and symptom severity score on presentation, injury characteristics, and balance assessment results were analyzed for each outcome group.

\section{Statistical Analysis}

The relationship between potential risk factors and recovery outcome status was assessed with both univariate and multivariate methods. For categorical variables, chisquare tests and Fisher exact test, where appropriate, were used to determine the statistical significance of any differences according to recovery status. Categorical variables included sex; presence or absence of SCAT2 score assessment; and a history of conditions, including attention deficit hyperactivity disorder (ADHD), migraines, previous concussion, amnesia, loss of consciousness, and balance problems. Two-sample independent t-tests were used to assess significance of differences in the continuous variables age, number of previous concussions, total SCAT2 score, and SCAT2 symptom severity score.

Simple logistic regression was used to identify independent associations with delayed recovery outcome and unadjusted odds ratio and $95 \%$ confidence intervals were calculated for each predictor variable. Covariates with a $\mathrm{p}$ $<0.2$ on univariate analysis were considered for inclusion in evaluating multivariate models, with the exception of total SCAT2 score and number of previous concussions, which were excluded because they exhibited colinear relationships with a SCAT2 score less than 80 and with a history of previous concussion, respectively.

Because the SCAT2 score was strongly associated with delayed recovery in patients who were given this assessment tool but SCAT2 scoring was obtained for only 57\% of the study cohort, we were concerned that pooling data from patients with and without SCAT2 scores would bias the predictive models. The potential for effect modification was assessed, and this assessment confirmed a sig- 
nificant association of delayed recovery with SCAT2 score availability; potential covariates were therefore stratified by presence or absence of a SCAT2 score assessment. Multivariate logistic regression was used to estimate separately adjusted stratum-specific ORs for delayed recovery outcomes among patients with SCAT2 scores and among patients without SCAT2 scores. All statistical analyses were performed with JMP Version 11.0 and SAS Version 9.3 (SAS Institute).

\section{Results}

Between August 2011 and January 2013, in total 562 patients had a concussion and presented to CCCOA. Sixtyseven of these patients presented more than 4 weeks after having his or her initial concussion, and 198 patients did not follow-up with CCCOA until symptom resolution was reported. These 265 patients were excluded from further analysis. Three additional patients who were older than 18 years were also excluded from this study. The remaining 294 patients were included in the case-control analysis. The control group, consisting of patients whose symptoms resolved within 28 days, had 189 patients (64\%), and the case group, comprising patients whose symptoms did not resolve within 28 days, had 105 patients (36\%) (Table 1).

\section{Baseline Characteristics}

Total SCAT2 and SCAT2 symptom severity scores were missing for $43 \%$ and $82 \%$ of the study patients, respectively. Overall, the case and control groups did not statistically significantly differ in available SCAT2 scores $(p=0.08)$. However, because the $p$ value of the latter analysis almost reached statistical significance and because we were concerned that the patients without SCAT2 scores might have had characteristics affecting delayed recovery, the effect of an available SCAT2 score on recovery was analyzed. This additional analysis showed that the estimates of the effects of potential risk factors on delayed recovery were significantly affected by whether or not the patient had received a SCAT2 score. Because the SCAT2 score was an effect modifier, all descriptive and inferential analyses were conducted separately for patients with and without SCAT2 scores.

The baseline characteristics of the patients with and without SCAT2 scores are presented in Table 1. The age of the patients in the SCAT2-score group ranged from 6 to 18 years (mean 13.7 years) and in the group without SCAT2 scores, from 4 to 18 years (mean 12.6 years), and this difference was not statistically significant $(\mathrm{p}=0.7)$. Among the patients who had undergone a SCAT2 assessment, delayed recovery was statistically significantly associated with female sex $(p=0.001)$, a history of previous concussion(s) $(\mathrm{p}=0.006)$, a SCAT2 score of less than 80 $(\mathrm{p}<0.0001)$, loss of consciousness at the time of injury $(\mathrm{p}=0.04)$, a lower initial total SCAT2 score $(\mathrm{p}<0.0001)$, a higher presenting SCAT2 symptom severity score $(\mathrm{p}<$ $0.0001)$, and incurring the concussion in a nonhelmet sport $(\mathrm{p}=0.008)$. In contrast, among patients without SCAT2 assessments, only female sex was significantly associated with delayed recovery $(\mathrm{p}=0.001)$, and the association of delayed recovery with participation in a nonhelmet sport was borderline statistically significant $(p=0.05)$.

\section{Recovery Differences Between SCAT2 Score Groups}

Among both the SCAT2-score and no-SCAT2-score

TABLE 1. Baseline characteristics stratified by SCAT2 score availability and recovery status*

\begin{tabular}{|c|c|c|c|c|c|c|c|c|}
\hline \multirow[b]{2}{*}{ Variable } & \multicolumn{4}{|c|}{ Patients w/ SCAT2 Scores } & \multicolumn{4}{|c|}{ Patients w/o SCAT2 Scores } \\
\hline & $\begin{array}{l}\text { All Patients } \\
(\mathrm{n}=168)\end{array}$ & $\begin{array}{l}\text { Controls } \\
\text { w/ Early } \\
\text { Recovery } \\
(n=115)\end{array}$ & $\begin{array}{l}\text { Cases w/ } \\
\text { Delayed } \\
\text { Recovery } \\
(n=53)\end{array}$ & p Value & $\begin{array}{l}\text { All Patients } \\
(n=126)\end{array}$ & $\begin{array}{c}\text { Controls } \\
\text { w/ Early } \\
\text { Recovery } \\
(n=74)\end{array}$ & $\begin{array}{c}\text { Cases w/ } \\
\text { Delayed } \\
\text { Recovery } \\
(n=52)\end{array}$ & $\mathrm{p}$ Value \\
\hline Mean age \pm SD & $13.7 \pm 2.5$ & $13.7 \pm 2.5$ & $13.8 \pm 2.5$ & 0.77 & $12.6 \pm 3.1$ & $12.6 \pm 3.1$ & $12.8 \pm 2.9$ & 0.44 \\
\hline \multicolumn{9}{|l|}{ No. of patients (\%) } \\
\hline Female sex & $39(23.2)$ & $20(17.4)$ & $19(35.8)$ & 0.001 & $38(30.2)$ & $14(18.9)$ & $24(46.2)$ & 0.001 \\
\hline $\mathrm{Hx}$ of $\mathrm{ADHD}$ & $14(8.3)$ & $7(6.1)$ & $7(13.2)$ & 0.14 & $13(10.3)$ & $5(6.8)$ & $8(15.4)$ & 0.12 \\
\hline $\mathrm{Hx}$ of migraines & $8(4.8)$ & $6(5.2)$ & $2(3.8)$ & 1.00 & $6(4.8)$ & $1(1.4)$ & $5(9.6)$ & 0.08 \\
\hline Previous concussion & $39(23.2)$ & $20(17.4)$ & $19(35.8)$ & 0.006 & $33(26.2)$ & $17(23.0)$ & $16(30.8)$ & 0.37 \\
\hline Loss of consciousness & $43(25.6)$ & $24(20.9)$ & $19(35.8)$ & 0.04 & $28(22.2)$ & $17(23.0)$ & $11(21.2)$ & 0.78 \\
\hline Loss of balance & $7(4.2)$ & $5(4.4)$ & $2(3.8)$ & 1.00 & $5(4.0)$ & $1(1.4)$ & $4(7.7)$ & 0.16 \\
\hline Nonhelmet sport $†$ & $57(37.5)$ & $32(30.5)$ & $25(53.2)$ & 0.008 & $50(46.7)$ & $24(38.7)$ & $26(57.8)$ & 0.05 \\
\hline \multicolumn{9}{|l|}{ Mean \pm SD } \\
\hline No. of previous concussions & $0.4 \pm 0.9$ & $0.3 \pm 0.7$ & $0.7 \pm 1.2$ & 0.03 & $0.3 \pm 0.7$ & $0.3 \pm 0.6$ & $0.4 \pm 0.7$ & 0.43 \\
\hline Total SCAT2 score & $81.2 \pm 10.9$ & $85.1 \pm 8.6$ & $72.9 \pm 10.9$ & $<0.0001$ & - & - & - & - \\
\hline SCAT2 score $<80$ & $69.8 \pm 7.8$ & $71.4 \pm 5.3$ & $67.9 \pm 8.9$ & $<0.0001$ & - & - & - & - \\
\hline SCAT2 symptom severity score & $18.0 \pm 20.1$ & $8.2 \pm 12.3$ & $35.2 \pm 19.7$ & $<0.0001$ & - & - & - & - \\
\hline
\end{tabular}


groups, most of the patients were boys $(77 \%$ and $70 \%$, respectively), and univariate logistic regression indicated that in both groups, female sex was associated with significantly greater odds of delayed recovery (Table 2). In addition, among the patients with SCAT2 scores, cases were also significantly more likely than controls to have reported a history of previous concussion, experienced loss of consciousness, or to have participated in a nonhelmet sport. Patients with SCAT2 scores less than 80 also had significantly greater odds of having a prolonged recovery than patients with higher SCAT2 scores. The univariate logistic regression also indicated that among patients without a SCAT2 assessment, no statistically significant differences in recovery existed between the case and control groups in having previous concussion, loss of consciousness, or other potential predictors shown in Table 2, except for the aforementioned higher risk for delayed recovery in girls.

\section{Effects of Sports Played}

Most of the patients incurred concussions during organized sports such as football, basketball, soccer, and cheerleading (Table 3). Of note, concussions acquired in a nonhelmet sport resulted in a higher risk for delayed recovery among both patients with and without SCAT2 scores. A statistically significant association of participation in a nonhelmet sport during the injury with delayed recovery was observed only among patients with SCAT2 scores (Table 2). The patients in the nonhelmet sport group reported loss of consciousness $(n=73)$, amnesia $(n=64)$, and balance difficulties $(n=12)$ after their concussions.

\section{Results of Multivariate Analysis}

Evaluation of all multivariate models that included female sex indicated that being female was consistently the strongest risk factor associated with postconcussive symptoms lasting longer than 28 days. The results of the final multivariate logistic models after stratification by presence or absence of SCAT2 scores are shown in Table 4. In the predictive model for patients with a SCAT2 assessment, female sex, a history of previous concussion, and SCAT2 score less than 80 remained significantly associated with delayed recovery. The multivariate analysis for predicting delayed recovery in patients without a SCAT2 assessment indicated that being female and having had a diagnosis of ADHD were the key risk factors associated with prolonged recovery from concussion.

\section{Discussion}

Concussions are a significant public health concern, and many states have passed legislation requiring all children that have a sports-related concussion to be evaluated by a physician. The physician is then required to medically clear the patient for return to play. Sports-related concussions are thus a unique medical entity in that the physician is required by law to provide prognostic information and take steps to medically clear the patient. The Alabama sports-related concussion law of 2011 requires that student athletes obtain medical clearance for return to play after a concussion and led to the development of the multidisciplinary concussion clinic at CCCOA. The concussion clinic was tasked with clearing patients for return to play over a large urban and rural area. To our knowledge, no previous case-control study has been conducted to help clinicians make key diagnostic and prognostic decisions in pediatric sports-related concussions.

The results of the present study show that female sex, a previous history of concussion(s), previously diagnosed ADHD, lower initial total SCAT2 score, higher presenting SCAT2 symptom severity score, and participation in a nonhelmet sport were all associated with a higher risk for postconcussive symptoms lasting more than 28 days. Other selected variables such as loss of consciousness, balance difficulties, and amnesia were not significantly associated with prolonged symptoms after a concussion. These findings provide useful prognostic information for patients and their parents, coaches, and athletic trainers. Additionally, these observations may result in a change in physician expectation of when a player may be allowed to return to play. The current recommendations require a

TABLE 2. Univariate logistic regression with potential predictors of delayed recovery for patients with or without SCAT2 scores*

\begin{tabular}{|c|c|c|c|c|c|c|}
\hline \multirow[b]{2}{*}{ Predictor } & \multicolumn{3}{|c|}{ Pts w/ SCAT2 Scores $(n=168)$} & \multicolumn{3}{|c|}{ Pts w/o SCAT2 Scores $(n=126)$} \\
\hline & No. of Pts & OR $(95 \% \mathrm{Cl})$ & $p$ Value & No. of Pts & OR $(95 \% \mathrm{Cl})$ & $\mathrm{p}$ Value \\
\hline Age & 168 & $1.02(0.90-1.16)$ & 0.76 & 126 & $0.96(0.85-1.07)$ & 0.44 \\
\hline Female sex & 168 & $2.65(1.27-5.56)$ & 0.01 & 126 & $3.67(1.66-8.15)$ & 0.001 \\
\hline$H x$ of ADHD & 168 & $2.35(0.78-7.07)$ & 0.15 & 126 & $2.51(0.77-8.16)$ & 0.13 \\
\hline $\mathrm{Hx}$ of migraines & 168 & $0.71(0.14-3.65)$ & 0.68 & 126 & $7.77(0.88-68.56)$ & 0.07 \\
\hline Previous concussion & 165 & $2.79(1.32-5.88)$ & 0.007 & 124 & $1.44(0.65-3.21)$ & 0.38 \\
\hline Total SCAT2 score & 168 & $0.89(0.85-0.92)$ & $<0.0001$ & - & - & - \\
\hline SCAT2 score $<80$ & 168 & $6.90(3.36-14.18)$ & $<0.0001$ & - & - & - \\
\hline Loss of consciousness & 167 & $2.10(1.02-4.30)$ & 0.04 & 125 & $0.88(0.37-2.09)$ & 0.79 \\
\hline Loss of balance & 168 & $0.86(0.16-4.60)$ & 0.86 & 126 & $3.08(0.66-56.09)$ & 0.11 \\
\hline Nonhelmet sport & 152 & $2.59(1.28-5.26)$ & 0.008 & 107 & $2.17(0.99-7.73)$ & 0.05 \\
\hline
\end{tabular}


TABLE 3. Sport played at the time of concussion for patients with or without SCAT2 scores stratified by symptom duration

\begin{tabular}{|c|c|c|c|c|}
\hline \multirow[b]{2}{*}{ Sport } & \multicolumn{2}{|c|}{ No. Patients w/ SCAT2 Scores (\%) } & \multicolumn{2}{|c|}{ No. of Patients w/o SCAT2 Scores (\%) } \\
\hline & Symptoms <28 Days & Symptoms $>28$ Days & Symptoms <28 Days & Symptoms $>28$ Days \\
\hline Football & $65(56.6)$ & $14(26.4)$ & $34(45.9)$ & $12(23.1)$ \\
\hline Basketball & $10(8.7)$ & $5(9.4)$ & $8(10.8)$ & $8(15.4)$ \\
\hline Soccer & $9(7.8)$ & $3(5.7)$ & $4(5.4)$ & $1(1.9)$ \\
\hline Cheerleading & $2(1.7)$ & $1(1.9)$ & $1(1.4)$ & $4(7.7)$ \\
\hline Other sports & $19(16.5)$ & $24(45.3)$ & $15(20.3)$ & $20(38.4)$ \\
\hline Unspecified sport & $10(8.7)$ & $6(11.3)$ & $12(16.2)$ & $7(13.5)$ \\
\hline Helmet sport & 73 (69.5) & $22(46.8)$ & 38 (61.3) & $19(42.2)$ \\
\hline Nonhelmet sport & $32(30.5)$ & $25(53.2)$ & $24(38.7)$ & $26(57.8)$ \\
\hline
\end{tabular}

graduated return-to-play protocol and specify a minimum of 1 week for return to play. Prospective studies of this pediatric population may indicate that certain patients could benefit from extended cognitive and physical rest period to improve the long-term outcomes of a concussion.

The study was designed to provide clinicians with predictors that could be used to determine if a pediatric patient with concussion is more likely to exhibit prolonged postconcussive symptoms. The only modifiable variable analyzed in this study was use of a helmet. We found that concussions acquired in a helmet-requiring sport lasted for a shorter time than those incurred in a nonhelmet sport. Whether this observation represented decreased concussion severity or merely reflected reporting bias (e.g., football players might be more likely to deny symptoms and return to play early than players in nonhelmet sports) remains unclear and merits further study.

Multiple studies have examined the relationship between age and recovery from concussion and have reported a longer postconcussive recovery time in younger than in older athletes. ${ }^{3,419}$ Our study was conducted at a pediatric hospital whose patients have a mean age of 13 years. Age within this pediatric population was not found to be associated with delayed recovery. Although some studies have suggested that concussive amnesia may predict an individual having postconcussive symptoms for longer than 28 days, our study reinforced another study's finding that amnesia is not associated with prolonged symptom duration. ${ }^{4,6,19}$
The present study has certain limitations that require further prospective study. The study was performed retrospectively, and initial documentation relied on patient and family recalls. The potential for selection bias is another possible concern because among the 562 patients presenting to CCCOA during the study period, $52.3 \%$ met all of the study eligibility criteria and were enrolled in the study. In addition, not all of our patients underwent SCAT2 and SCAT2 symptom severity score testing. A potential limitation of this study was that most participants were seen in a multidisciplinary clinic, and these findings may not be generalizable to other outpatient settings. An additional concern is that in a state like Alabama, where participation in sports, particularly in football, is associated with significant social pressure, patients may not be entirely honest in their reporting of symptoms, as such reporting will preclude them from returning to play.

\section{Conclusions}

In this case-control study, a history of previous concussion(s), a presenting SCAT2 score of less than 80, a history of ADHD, female sex, and playing a nonhelmet sport were all associated with a higher risk for prolonged recovery ( $>28$ days) after a concussion. Large prospective cohort studies are needed to definitively establish any associations between clinical predictors and prolonged recovery following concussion. Data from these studies may then be used to develop clinical prediction models to

TABLE 4. Multivariate logistic regression with predictors of delayed recovery for patients with or without SCAT2 scores*

\begin{tabular}{|c|c|c|c|c|}
\hline \multirow[b]{2}{*}{ Predictor } & \multicolumn{2}{|c|}{ Patients w/ SCAT2 Scores $(n=168)$} & \multicolumn{2}{|c|}{ Patients w/o SCAT2 Scores $(n=126)$} \\
\hline & $\mathrm{aOR}(95 \% \mathrm{Cl})$ & p Value & $\mathrm{aOR}(95 \% \mathrm{Cl})$ & $\mathrm{p}$ Value \\
\hline Female sex & $3.48(1.43-8.49)$ & 0.006 & $4.41(1.93-10.07)$ & 0.0004 \\
\hline $\mathrm{Hx}$ of ADHD† & - & - & $3.87(1.13-13.24)$ & 0.03 \\
\hline Previous concussion & $3.67(1.51-8.95)$ & 0.004 & - & - \\
\hline SCAT2 score $<80 \dagger$ & $5.58(2.61-11.93)$ & $<0.0001$ & - & - \\
\hline $\begin{array}{l}\text { aOR }=\text { adjusted } O R \text {. } \\
\text { * A p }<0.05 \text { was considered stat } \\
\text { † Dashes in this row indicate no } \\
\text { analysis. }\end{array}$ & $\begin{array}{l}\text { e final multivariate } m \\
\text { cant. }\end{array}$ & Ise no sta & ificance was detected & iate \\
\hline
\end{tabular}


further guide clinicians in the optimal treatment of youth athletes diagnosed with concussion.

\section{References}

1. Barlow KM, Crawford S, Stevenson A, Sandhu SS, Belanger F, Dewey D: Epidemiology of postconcussion syndrome in pediatric mild traumatic brain injury. Pediatrics 126:e374e381, 2010

2. Cancelliere C, Hincapié CA, Keightley M, Godbolt AK, Côté P, Kristman VL, et al: Systematic review of prognosis and return to play after sport concussion: results of the International Collaboration on Mild Traumatic Brain Injury Prognosis. Arch Phys Med Rehabil 95 (3 Suppl):S210-S229, 2014

3. Collins M, Lovell MR, Iverson GL, Ide T, Maroon J: Examining concussion rates and return to play in high school football players wearing newer helmet technology: a three-year prospective cohort study. Neurosurgery 58:275-286, 2006

4. Field M, Collins MW, Lovell MR, Maroon J: Does age play a role in recovery from sports-related concussion? A comparison of high school and collegiate athletes. J Pediatr 142:546-553, 2003

5. Gronwall D, Wrightson P: Cumulative effect of concussion. Lancet 2:995-997, 1975

6. Guskiewicz KM, McCrea M, Marshall SW, Cantu RC, Randolph C, Barr W, et al: Cumulative effects associated with recurrent concussion in collegiate football players: the NCAA Concussion Study. JAMA 290:2549-2555, 2003

7. Guskiewicz KM, Ross SE, Marshall SW: Postural stability and neuropsychological deficits after concussion in collegiate athletes. J Athl Train 36:263-273, 2001

8. Iverson GL, Brooks BL, Collins MW, Lovell MR: Tracking neuropsychological recovery following concussion in sport. Brain Inj 20:245-252, 2006

9. Johnson EW, Kegel NE, Collins MW: Neuropsychological assessment of sport-related concussion. Clin Sports Med 30:73-88, viii-ix, 2011

10. Kirkwood MW, Yeates KO, Wilson PE: Pediatric sportrelated concussion: a review of the clinical management of an oft-neglected population. Pediatrics 117:1359-1371, 2006

11. Lau BC, Kontos AP, Collins MW, Mucha A, Lovell MR: Which on-field signs/symptoms predict protracted recovery from sport-related concussion among high school football players? Am J Sports Med 39:2311-2318, 2011

12. Lau B, Lovell MR, Collins MW, Pardini J: Neurocognitive and symptom predictors of recovery in high school athletes. Clin J Sport Med 19:216-221, 2009

13. Lovell MR, Collins MW, Iverson GL, Field M, Maroon JC, Cantu R, et al: Recovery from mild concussion in high school athletes. J Neurosurg 98:296-301, 2003
14. McCrea M, Guskiewicz KM, Marshall SW, Barr W, Randolph C, Cantu RC, et al: Acute effects and recovery time following concussion in collegiate football players: the NCAA Concussion Study. JAMA 290:2556-2563, 2003

15. McCrory P, Meeuwisse WH, Aubry M, Cantu RC, Dvořák $\mathrm{J}$, Echemendia RJ, et al: Consensus statement on concussion in sport: the 4th International Conference on Concussion in Sport, Zurich, November 2012. J Athl Train 48:554-575, 2013

16. McCrory P, Meeuwisse W, Johnston K, Dvorak J, Aubry M, Molloy M, et al: Consensus statement on concussion in sport: the 3rd International Conference on Concussion in Sport held in Zurich, November 2008. J Athl Train 44:434-448, 2009

17. Meehan WP III, d'Hemecourt P, Collins CL, Comstock RD: Assessment and management of sport-related concussions in United States high schools. Am J Sports Med 39:23042310, 2011

18. Meehan WP III, Mannix RC, Stracciolini A, Elbin RJ, Collins MW: Symptom severity predicts prolonged recovery after sport-related concussion, but age and amnesia do not. J Pediatr 163:721-725, 2013

19. Pellman EJ, Lovell MR, Viano DC, Casson IR, Tucker AM: Concussion in professional football: neuropsychological testing-part 6. Neurosurgery 55:1290-1305, 2004

\section{Disclosures}

The authors report no conflict of interest concerning the materials or methods used in this study or the findings specified in this paper.

\section{Author Contributions}

Conception and design: Johnston, Miller. Acquisition of data: Johnston, Miller, Gill, O’Neill, Brown, Crowther, Ferguson. Analysis and interpretation of data: Johnston, Miller, Kuhn, Rocque. Drafting the article: Johnston, Miller, Kuhn, Rocque, Menendez, Crowther. Critically revising the article: Johnston, Miller, Kuhn, Rocque, Menendez, Crowther. Reviewed submitted version of manuscript: Johnston, Miller, Davis. Statistical analysis: Johnston, Miller, Agee. Study supervision: Johnston.

\section{Correspondence}

James M. Johnston, Department of Neurosurgery, University of Alabama at Birmingham, FOT 1030, 1720 2nd Ave. S, Birmingham, AL 35294. email: james.johnston@childrensal.org. 